\title{
ON THE CENTRAL MANAGEMENT OF RISK NETWORKS
}

\author{
FLORIN AVRAM, ${ }^{*}$ Université de Pau \\ ANDREEA MINCA, ${ }^{* *}$ Cornell University
}

\begin{abstract}
In this paper we identify three questions concerning the management of risk networks with a central branch, which may be solved using the extensive machinery available for one-dimensional risk models. First, we propose a criterion for judging whether a subsidiary is viable by its readiness to pay dividends to the central branch, as reflected by the optimality of the zero-level dividend barrier. Next, for a deterministic central branch which must bailout a single subsidiary each time its surplus becomes negative, we determine the optimal bailout policy, as well as the ruin probability and other risk measures, in closed form. Moreover, we extend these results to the case of hierarchical networks. Finally, for nondeterministic central branches with one subsidiary, we compute approximate risk measures by applying rational approximations, and by using the recently developed matrix scale methodology.
\end{abstract}

Keywords: Multidimensional risk process; Sparre Andersen process; Markov additive process; matrix exponential approximation; ruin probability

2010 Mathematics Subject Classification: Primary 60G51

Secondary 60K30; 60J75

\section{Introduction}

In recent years considerable effort has been devoted in the finance and insurance literature to model default (or ruin) in the context of a system of interacting firms. The insurance giant American International Group, inc. (AIG) was almost led to bankruptcy (and itself received a bailout which topped $\$ 180$ billion from the US government) after its subsidiary AIG Financial Products faced contingent claims on its derivatives positions and forced a massive government bailout in 2008. AIG Financial Products, liquidated in 2011, will probably remain as a textbook example of a nonviable subsidiary whose losses were disproportionate with respect to its prior profitability.

Our object of interest in this paper is a general model of networks with a central branch which needs to bail out its subsidiaries. An analog in the current economy is the central clearing counterparty, mandated by the Dodd-Frank Wall Street Reform and Consumer Protection Act in response to the recent crisis. Just as a central branch can be ruined by one subsidiary, a central clearing house can default if its capital is insufficient to cover the default of one (or several) of its members. This in itself could be a systemic event, so it is a current concern if central counterparties are effectively managed.

Received 10 November 2015; revision received 18 March 2016.

* Postal address: Département de Mathématiques, Université de Pau, Pau 64000, France.

Email address: florin.avram@univ-pau.fr

** Postal address: School of Operations Research and Information Engineering, Cornell University, Ithaca,

NY 14850, USA. Email address: acm299@cornell.edu 
Another example which falls under the umbrella of a central branch is a reinsurance company which could face potentially large claims, depending on the primary insurers' deficit. For example in the year 2011, the amount of global insured losses was around $\$ 110$ billion and over half of this amount was reinsured [33]. While these are not bailouts, the principle is the same: one reinsurer can insure several insurers for their losses (in exchange for the transfer of part of their stream of insurance fees). The idea of reinsurance is risk sharing: insurers are ideally independent, so the reinsurer can diversify risk. In many cases, one large reinsurer has several business lines, e.g., property and casualty, life insurance, health insurance, etc. We will refer generically to a subsidiary as a subunit of the central branch and in practice it can also be a business line.

In all these cases it is important for the central branch to first decide which subsidiary is viable, and second, how to optimally manage the central branch in presence of subsidiaries.

\subsection{From one to multiple dimensions}

Multidimensional first-passage problems are considerably more difficult than one-dimensional ones, and one cannot expect general formulas. One exception is a Pollaczek-Khinchinetype formula for the transform of ruin probabilities $\Psi(\boldsymbol{u})$ of spectrally negative networks provided in the foundational paper [15]. However, this formula involves several unknown functions (the Laplace transforms over each boundary facet of the state space), and it is not at all obvious how to exploit this formula numerically. In this paper we provide approximations in the multidimensional case by reduction to one-dimensional results.

A key point concerning central branch networks is defining a notion of the 'profitability' of a subsidiary for the ensemble of the business. Whereas intuitively it is clear that not all subsidiaries represent viable businesses from the perspective of the central branch, and some should be closed, it is far from clear which optimization criteria should be utilized to achieve that. The criterion we propose below is to judge spectrally negative Lévy subsidiaries by the optimality of the zero-level dividend barrier policy, a criterion which could be called call efficiency or readiness. The motivation is that this combines simplicity of calculation with a complete utilization of the law of the process, and that it reflects an arguably important characteristic to be expected from a member of a coalition.

\subsection{Contents and contributions}

We start with an introduction to risk networks in Section 2 and some background on onedimensional first-passage problems in Section 3.

Our first contribution, in Section 4, is to explore several possible definitions of efficiency, before settling on one which is shown to provide reasonable results for a wide variety of subsidiaries satisfying the conditions of Theorem 1, which include exponential claims.

Our second contribution, Theorem 2, applies to a central branch with purely deterministic cash inflows with one subsidiary. In this case, the computation of the finite-time ruin probabilities and other performance measures (including total subsidiary dividends until ruin) reduces to the corresponding computation for a subsidiary with modified initial capital and income rate. For other potentially useful explicit computations, see [9], [13]. In particular, this reduction result applies also for the value function of the de Finetti or other optimization objectives; see Remark 8, without any distributional assumptions. For example, the subsidiaries may be dual risk processes, or spectrally two-sided Lévy processes.

The proof of this result, via a pathwise argument, yields also an upper bound when $I>1$, and an extension to hierarchical networks is given in Corollary 1. The notion of hierarchical 
networks, introduced in Gerber [20], has been used to model chains of reinsurers and is the most important application of the deterministic central branch case.

Our third contribution, in Section 6.1, deals with nondeterministic central branches with one subsidiary, which do not admit an exact solution due to their complex dependent Sparre Andersen structure. We isolate the central branch and one subsidiary, and allow for a nondeterministic component of the central branch process to represent other claims of the central branch, for example, aggregate net flows of the other subsidiaries or other liquidity needs of the central branch. We propose an approximation approach based on the classic idea of rational approximations to replace the Sparre Andersen structure by a Markov modulated Lévy structure, and by subsequently using the matrix scale methodology.

We propose to obtain spectrally negative Markov additive process (SNMAP) approximations for non-MAP central branches, by using bivariate phase-type approximations for the joint law of the downward ladder time and height. The advantage of this approach is that once a SNMAP approximation is obtained, many similar problems may be solved just by applying the scale matrix methodology developed in [21], [23], [26], and using the SNMAP MATHEMATica ${ }^{\circledR}$ package of Ivanovs [22]. Different problems are thus solved simultaneously.

A numeric illustration is performed in Section 6.2, where we consider the problem of choosing a barrier $B$ maximizing central branch dividends until ruin, in the case of one subsidiary with exponential claims (and without dividends). In this case, univariate phase-type approximations of the downward ladder density, obtained via a continued fraction expansion (see Section 6.1), provide a SNMAP approximation of the central branch process. Note that even though here the central branch claim arrival times have an explicit Bessel-type law, our approach replaces this by a phase-type approximation. Subsequently, using the SNMAP package from [22] provides the optimal barrier.

\section{Some background on risk networks}

Multidimensional risk networks (MRNs) are defined by

$$
\boldsymbol{X}(t)=\boldsymbol{u}+\boldsymbol{c} t-\boldsymbol{S}(t)=\left(X_{i}(t), t \geq 0, i \in \mathcal{L}\right),
$$

where $\mathcal{I}=[1, \ldots, I]$ is a finite set, the vector $\boldsymbol{u}$ represents the capital of the MRN at time 0 , the vector $c$ represents a constant cash inflow rate, and $\boldsymbol{S}(t)$ is a process representing cash outflows at time $t$, which may include both Lévy and Sparre Andersen renewal components. If no boundary condition is specified, we will call this a free spectrally negative MRN.

Remark 1. The minus sign comes from the one-dimensional case most studied historically, the spectrally negative Cramér-Lundberg process, but the case when $X(t)$ is spectrally positive is also interesting. The case of spectrally two-sided $X(t)$ is of course interesting, but more challenging.

Remark 2. The simplest case is when the components are independent and identically distributed (i.i.d.) compound renewal Sparre Andersen processes, generated by i.i.d. pairs of interarrival times and claims $\left(A_{j}^{(i)}, C_{j}^{(i)}\right), j=1,2, \ldots$,

$$
X_{i}(t)=u_{i}+c_{i} t-S_{i}(t), \quad S_{i}(t)=\sum_{j=1}^{N_{i}(t)} C_{j}^{(i)}, \quad i=1, \ldots, I,
$$

where $N_{i}(t)=\max \left\{k: T_{k}^{(i)}:=\sum_{j=1}^{k} A_{j}^{(i)} \leq t\right\}$ are renewal counting processes associated to the independent interarrivals (see, e.g. [4]), with intensity $\lambda_{i}=\mathbb{E}\left(A_{1}^{(i)}\right)^{-1}$, and the claim 
sizes $C_{j}^{(i)}, j \geq 1$ are nonnegative i.i.d. random variables with arbitrary marginal distribution functions denoted by $F_{i}(x), i \in \mathcal{L}$, with finite expectation, denoted by $m_{i}$. However, the restriction to jump processes is not essential.

Remark 3. After reaching special subsets exterior to the state space, several continuation/regulation mechanisms are possible, such as absorption, reflection, or jumping to the interior. These correspond to various possible interactions between the components at times of distress.

Example 1. (A toy example with one absorbing boundary and several reflecting boundaries.) Consider a central branch which must simultaneously manage several subsidiaries. The central branch will keep the subsidiaries solvent by bailouts until the moment of its bankruptcy, or according to a prespecified rule, e.g. until the subsidiary is deemed nonprofitable. The subsidiaries will pay dividends to the central branch. Finally, the expected present (net) value to the central branch of a subsidiary consists in the difference between the expected discounted payments and bailout amounts.

This example suggests the following model.

Definition 1. A central branch (CB) network is formed from the following constituents.

- A unit, called a $\mathrm{CB}$, whose ruin causes the ruin of the whole network.

- Several subsidiaries $X_{i}(t), i=1, \ldots, I$ that must be kept nonnegative or above certain prescribed levels, by transfers from the CB.

For this network, the boundaries $u_{i}=0, i=1, \ldots, I$ are reflecting and $u_{0}=0$ is absorbing.

Remark 4. There are many applications of the CB concept: a government/central bank, a reinsurance company, an insurance group, a central clearing house, etc. Another interesting application is that of a coalition or default fund created by several institutions, for bailing them out when they go bankrupt. Interesting issues here are determining fair conditions for merging into (profit participation schemes) and splitting out of the coalition.

One of the most important activities of CBs is acquiring and closing subsidiaries. A critical building block in understanding the management of the $\mathrm{CB}$ risk network is modeling one subsidiary managed by the $\mathrm{CB}$. The benefit is represented by the dividends received from the subsidiary, while the costs are the cumulated bail-out costs, the number of bailouts, up to a certain time set by the CB and referred to as 'patience'. Establishing decoupled rules for the management of subsidiaries is the key step towards solving problems pertaining to the whole risk network, such as maximizing the total cumulated net income from all the subsidiaries, until the eventual bankruptcy of the CB.

In the sequel we will use the following notation.

Notation. Denote the ruin times and ruin probabilities (finite time and eventual) of each subsidiary $X_{i}(t), i=1,2, \ldots$ when isolated from the network by

$\tau_{i}\left(u_{i}\right)=\inf \left\{t \geq 0: X_{i}(t)<0\right\}, \quad \Psi_{i}\left(t, u_{i}\right)=\mathbb{P}\left(\tau_{i}\left(u_{i}\right)<t\right), \quad \Psi_{i}\left(u_{i}\right):=\mathbb{P}\left(\tau_{i}\left(u_{i}\right)<\infty\right)$,

and let

$$
\tau_{0}=\inf \left\{t \geq 0: X_{0}(t)<0\right\}
$$


denote the ruin time of the $\mathrm{CB} X_{0}(t)$. The ruin probability of the $\mathrm{CB}$ and its Laplace transform will be denoted respectively by

$$
\Psi(t, \boldsymbol{u})=\Psi(t, \boldsymbol{u}, \boldsymbol{c})=\mathbb{P} \boldsymbol{u}\left[\tau_{0}<t\right], \quad \widehat{\psi}_{q}(\boldsymbol{u}):=\widehat{\psi}_{q}(\boldsymbol{u}, \boldsymbol{c})=\mathbb{E}_{\boldsymbol{u}}\left[\mathrm{e}^{-q \tau_{0}}\right],
$$

where $\boldsymbol{u}=\left(u_{0}, u_{1}, \ldots\right), \boldsymbol{c}=\left(c_{0}, c_{1}, \ldots\right)$ are the initial reserves and income rates of the network components.

\section{Preliminaries on first passage}

Consider a spectrally negative MAP $(X(t), J(t))$ and assume that none of the underlying Lévy processes $X_{t}^{i}$ is almost surely nonincreasing, see [3], [21] for more information on these processes. Define the first-passage times by

$$
\tau_{b}^{+}=\inf \{t \geq 0: X(t)>b\}, \quad \tau_{b}^{-}=\inf \{t \geq 0: X(t)<b\},
$$

with inf $\varnothing=+\infty$. We will sometimes write $\tau$ for the 'ruin time' $\tau_{0}^{-}$.

Let $\tau_{a, b}=\tau_{a}^{-} \wedge \tau_{b}^{+}$denote the 'two-sided' exit time from an interval $[a, b]$.

The classic De Finetti objective maximizes expected discounted dividends until the ruin time. For judging efficiency, it is natural to take also into account a final bail-out, resulting in the optimization objective

$$
V^{(w)}(x)=\sup _{\pi} \mathbb{E}_{x}\left[\int_{0}^{\tau} \mathrm{e}^{-q t} \mathrm{~d} D^{\pi}(t)+\mathrm{e}^{-q \tau} w(U(\tau))\right],
$$

where $w(u)$ is a so-called Gerber-Shiu penalty function.

The optimal dividend distribution is of multibarrier type [19], and the end result may be expressed in terms of scale functions [8], [11]. Further conditions are necessary to ensure that single constant barrier strategies suffice [8], [12], [28], [29].

We judge efficiency of subsidiaries over an infinite horizon by optimizing bailouts and dividends. Over an infinite horizon, the subsidiary will possibly need to be bailed out a number $N_{B} \geq 0$ of times.

In the case of linear transaction costs $k u-K$, the optimization objective (of particular interest in a bailout setting) becomes the expectation over an infinite horizon of a linear combination of discounted dividends $D(t)$, cumulative bailouts $Z(t)$, and number of bailouts $N_{B}^{\pi}(t)$ up to time $t$, that is,

$$
V^{(k)}(x)=\sup _{\pi} \mathbb{E}_{x}\left[\int_{0}^{\infty} \mathrm{e}^{-q t} \mathrm{~d} D^{\pi}(t)-k \int_{0}^{\infty} \mathrm{e}^{-q t} \mathrm{~d} Z^{\pi}(t)-K \int_{0}^{\infty} \mathrm{e}^{-q t} \mathrm{~d} N_{B}^{\pi}(t)\right] .
$$

Since in a diffusion setting this objective was first considered by Shreve, Lehoczky, and Gaver [32]—see also Lokka and Zervos [30]—we will call it the SLG objective.

For spectrally negative Lévy processes, the optimal dividend distribution for the SLG objective is always of constant barrier type, and the end result may be expressed in terms of scale functions [8].

\section{Efficient subsidiaries}

Efficient subsidiaries. A crucial issue for a coalition is how to accept efficient members and eventually reject them if they are not efficient. For that it is natural to evaluate each member separately, by classic one-dimensional risk measures such as ruin probabilities, or the value of future dividend payments made to the coalition. However, the choice of an economic principle for evaluating efficiency is not at all obvious. 
One often used approach is to optimize discounted dividends of rate $d=c \gamma, \gamma \in(0,1]$ taken above a constant threshold $b$ ( $\gamma$ represents the proportion of income taken above the threshold); see, e.g. [2], [5]. The process obtained by subtracting the dividends is called reflected when $\gamma=1$ and refracted when $\gamma \in(0,1)$.

We now examine some efficiency concepts based on prior literature.

Example 2. (A simple, but unsatisfactory definition of efficiency.) Consider defining efficiency as nonnegativity of the SLG value function for the 0 barrier, when dividends are $D(t)=d t$. By [8, Theorem 1, Equation (4.4)],

$$
V^{(k)}(0)=\frac{c}{q}-k \frac{\lambda m_{1}}{q} \geq 0
$$

yields $k \leq c / \lambda m_{1}=\rho^{-1}$. This generalizes easily for the 0 threshold, yielding

$$
k \leq \frac{d}{\lambda m_{1}}=\rho^{-1} \gamma .
$$

Example 3. (SLG readiness.) Consider now efficiency defined as the optimality of $b=0$ for the SLG objective with constant reflecting barrier. This problem was fully analyzed in $[8$, Theorem 3], and, in particular, from [8, Lemma 2] it was shown that the optimal SLG constant barrier is $b^{*}=0$ if and only if $k \leq 1+q / \lambda$.

By this criterion, a subsidiary $i$ with

$$
k_{i} \leq 1+\frac{q}{\lambda_{i}}
$$

will be deemed totally efficient and accepted for ever in the coalition. Otherwise (see [8, Equation (5.6)]) $b^{*}=\inf \left\{a>0:\left[k Z^{(q)}(a)-1\right] W^{(q) \prime}(a)-k q W^{(q)}(a)^{2} \leq 0\right\}>0$ (i.e. the subsidiary must be forgiven from paying dividends in between $\left(0, b^{*}\right)$. See also [24], [18] for related results. Subsidiaries with loading condition $\rho_{i}<1$ and

$$
k_{i}>1+\frac{q}{\lambda_{i}}
$$

will be deemed partially efficient and accepted only for a random time with law $\mathcal{E}\left(\theta_{i}\right)$, where $\theta_{i}+q=\lambda_{i}\left(k_{i}-1\right)$ (rendering thus $b_{i}^{*}=0$ optimal with respect to the total discount rate $\left.\delta_{i}=q+\theta_{i}\right)$.

Unfortunately, the criterion (1) does not take into account the claim size law.

To avoid these shortcomings, we introduce now a new efficiency concept.

Judging efficiency as readiness to pay dividends. Another method to judge efficiency was made in paper [6], whose authors proposed to define efficiency as the local optimality of $b=0$ over some interval $[0, \varepsilon), \varepsilon>0$.

The motivation is that such subsidiaries are functional from the start and can contribute cash flows to the $\mathrm{CB}$ without having to wait first until its reserves build out; effectiveness is thus translated in this paper as readiness. A second motivation is that inefficient subsidiaries may be turned into efficient subsidiaries by setting up rules to monitor their time passed in 'orange zones', and eventually close them when necessary. This may be achieved by 'killing' them with a rate $\theta_{i}$ in the orange zone, where $\theta_{i}$ is chosen to render the barrier $b=0$ locally optimal. Furthermore, one may use 'two step' killing rates, and choose the killing rate as $\infty$ in 'red zones', as suggested in [17]. We show in a variety of examples that this procedure produces reasonable results. 
In conclusion, we postulate that a subsidiary has either of the following properties.

- Nonefficient and rejected immediately if its loading factor $c / \lambda \mathbb{E}\left[C_{1}\right]-1:=\rho^{-1}-1$ is not nonnegative, since this implies an infinite number of bailouts.

- Totally efficient and accepted for ever in the coalition if and only if

$$
\rho=\lambda \frac{\mathbb{E}\left[C_{1}\right]}{c}<1 \quad \text {, and } k \leq f(q),
$$

where $q$ is the discount rate, and $f(q)$ is an increasing function of $q$, with $f(q)<0$, obtained as optimality of $b=0$ for some specific dividends distribution scheme, and $k \geq 1$ captures the cost associated with capital infusions towards a subsidiary.

- Partially efficient if the loading condition $\rho<1$ is satisfied, and $k>f(q)$. These subsidiaries will also be accepted, but only with an impatience rate $\theta$, resulting in killing the subsidiary after its time or lowest value in an orange zone exceeds an exponential random variable of rate $\theta$. The impatience rate $\theta$ is chosen so that

$$
\tilde{f}(q, \theta)=k,
$$

where $\tilde{f}(q, \theta)$ is computed from the optimality of $b=0$ for the impatience modified value function. This is illustrated in Example 3 below, where $\tilde{f}(q, \theta)=f(q+\theta)$.

We turn now to a new possible efficiency criterion.

\subsection{Efficiency based on the de Finetti constant dividend barrier and linear penalty}

As noted above, a critical step in defining efficiency is the choice of the value function. Our choice is based on the case of the de Finetti constant barrier and linear penalty (with $k$ proportional cost and $K$ the fixed cost), for which the value function is known explicitly, see [10, Equation (13)], [11], and [29], in terms of the $W$-scale function [14], and of the $Z$-scale function [7] defined by

$$
Z_{q}(x):=1+q \bar{W}_{q}(x), \quad \bar{W}_{q}(x):=\int_{0}^{x_{+}} W_{q}(y) \mathrm{d} y .
$$

Putting $\bar{F}(x)=\int_{0}^{x} F(u) \mathrm{d} u, F(u)=k\left(Z(u)-\kappa^{\prime}(0) W(u)\right)-K q W(u)$, the value function may be written as

$$
\begin{gathered}
V(x)=\bar{F}(x)+W(x) G(b), \\
G(b)=\frac{1-F(b)}{W^{\prime}(b)}=\frac{1-k\left(Z(b)-\kappa^{\prime}(0) W(b)\right)+K q W(b)}{W^{\prime}(b)} .
\end{gathered}
$$

The function $F(u)=-K Z^{(0)}(u)+k Z^{(1)}(u)$, where the coefficients of $-K, k$ in $F(x)$ are found by differentiating the second scale function [11] $Z(x, \theta)=\mathrm{e}^{\theta x}\left(1-\kappa(\theta) \int_{0}^{x} \mathrm{e}^{-\theta y} W(y) \mathrm{d} y\right)$, 0 and 1 times, respectively, with respect to $\theta$, and then differentiating once more with respect to $x$.

The optimality condition is obtained by differentiating the 'barrier influence function' $G(b)$ $\left[11\right.$, Section 13.1]. Letting $\widetilde{q}:=q / c, \Delta(b)=c\left(W^{\prime 2}(b)-W(b) W^{\prime \prime}(b)\right)$, we find that

$$
\begin{aligned}
H(b):= & G^{\prime}(b)\left(W^{\prime}(b)\right)^{2} \\
= & \left.\left(k\left(\kappa^{\prime}(0) W^{\prime}(b)-q W(b)\right)\right)+K q W^{\prime}(b)\right) W^{\prime}(b) \\
& -W^{\prime \prime}(b)\left(1+k\left(\kappa^{\prime}(0) W(b)-1-q \bar{W}(b)\right)+K q W(b)\right)
\end{aligned}
$$




$$
\begin{aligned}
= & (K \tilde{q}+k(1-\rho)) c\left(W^{\prime 2}(b)-W(b) W^{\prime \prime}(b)\right) \\
& +k q\left(W^{\prime \prime}(b) \bar{W}(b)-W^{\prime}(b) W(b)\right)+(k-1) W^{\prime \prime}(b) \\
= & k\left((1-\rho) \Delta(b)+q\left(W^{\prime \prime}(b) \bar{W}(b)-W^{\prime}(b) W(b)\right)+W^{\prime \prime}(b)\right) \\
& +K \widetilde{q} \Delta(b)-W^{\prime \prime}(b) .
\end{aligned}
$$

Now in order to compute $W^{\prime \prime}(0)$, let

$$
K(s)=\frac{1}{c} \frac{\tilde{\lambda} s \widehat{\bar{F}}(s)}{1-\tilde{\lambda} \widehat{\bar{F}}(s)-\delta / s}, \quad \tilde{\lambda}:=\frac{\lambda}{c},
$$

the expression used in [25, p. 33] to find $W^{\prime}(0)=\lim _{s \rightarrow \infty} K(s)=(\tilde{\lambda}+\tilde{q}) / c$. Following the same approach, we obtain

$$
W^{\prime \prime}(0)=\lim _{s \rightarrow \infty} s\left(K(s)-W^{\prime}(0)\right)=\frac{1}{c}\left((\tilde{\lambda}+\tilde{q})^{2}-\tilde{\lambda} f_{C}(0)\right) .
$$

Furthermore, using $\bar{W}(0)=0, W(0)=1 / c$, and $c \Delta(0)=\tilde{\lambda} f_{C}(0)$, we have

$$
\begin{aligned}
c G^{\prime}(0)\left(W^{\prime}(0)\right)^{2} & =k\left((1-\rho) \tilde{\lambda} f_{C}(0)-q W^{\prime}(0)+c W^{\prime \prime}(0)\right)+K \tilde{q} \tilde{\lambda} f_{C}(0)-c W^{\prime \prime}(0) \\
& =k\left(\tilde{\lambda}(\tilde{\lambda}+\tilde{q})-\rho \tilde{\lambda} f_{C}(0)\right)+K \tilde{q} \tilde{\lambda} f_{C}(0)-\left((\tilde{\lambda}+\tilde{q})^{2}-\tilde{\lambda} f_{C}(0)\right) .
\end{aligned}
$$

The optimality condition is

$$
G^{\prime}(0) \leq 0 \quad \Longleftrightarrow \quad k \leq f(\widetilde{q}, K):=\tilde{\lambda}^{-1} \frac{(\tilde{\lambda}+\tilde{q})^{2}-\tilde{\lambda} f_{C}(0)-K \tilde{q} \tilde{\lambda} f_{C}(0)}{\tilde{q}+\tilde{\lambda}\left(1-m_{1} f_{C}(0)\right)} .
$$

Remark 5. Note that if $K=0, f_{C}(0)=0$, this reduces to SLG readiness.

Example 4. As a check, with exponential claims, the scale function is

$$
W^{(q)}(x)=A_{+} \mathrm{e}^{\zeta^{+}(q) x}-A_{-} \mathrm{e}^{\zeta^{-}(q) x},
$$

where $A_{ \pm}=c^{-1}\left(\mu+\zeta^{ \pm}(q)\right) /\left(\zeta^{+}(q)-\zeta^{-}(q)\right)$, and $\zeta^{+}(q)=\Phi(q), \zeta^{-}(q)$ are the largest and smallest roots of the polynomial $c^{-1}(\psi(s)-q)(s+\mu)=s^{2}-s(\tilde{\lambda}+\tilde{q}-\mu)-\widetilde{q} \mu$, that is,

$$
\zeta^{ \pm}(q)=\frac{\tilde{q}+\tilde{\lambda}-\mu \pm \sqrt{(\widetilde{q}+\tilde{\lambda}-\mu)^{2}+4 \tilde{q} \mu}}{2} .
$$

Thus,

$$
\begin{aligned}
W^{\prime \prime}(0) & =A_{+}\left(\zeta^{+}(q)\right)^{2}-A_{-}\left(\zeta^{-}(q)\right)^{2} \\
& =\tilde{q} \mu\left(A_{+}-A_{-}\right)+(\tilde{\lambda}+\tilde{q}-\mu)\left(A_{+} \zeta^{+}(q)-A_{-} \zeta^{-}(q)\right)
\end{aligned}
$$

and

$$
c W^{\prime \prime}(0)=\tilde{q} \mu+(\tilde{\lambda}+\tilde{q}-\mu)(\tilde{\lambda}+\widetilde{q})=(\tilde{\lambda}+\widetilde{q})^{2}-\tilde{\lambda} \mu
$$

confirming (2).

Finally, with $K=0$ and exponential claims of rate $\mu$, we recover [11, Lemma 13.2(b)], that is,

$$
k \leq \frac{(\tilde{\lambda}+\tilde{q})^{2}-\tilde{\lambda} \mu}{\tilde{\lambda} \tilde{q}}=\frac{(\lambda+q)^{2}-\lambda c \mu}{\lambda q} .
$$


Lemma 1. With general claims admitting a first moment such that $\rho \leq m_{1} f_{C}(0) \leq 1$, the function $f(\widetilde{q}, K), \widetilde{q}>0$, defined in (3) is increasing for any

$$
0 \leq K \leq \frac{\rho^{-1}-1}{1-m_{1} f_{C}(0)}+\frac{1}{m_{1} f_{C}(0)}
$$

Proof. The function defined in the right-hand side of (3) is eventually increasing when $q \rightarrow \infty$. Its value at 0 is proportional to $\left(\rho-m_{1} f_{C}(0)\right) /\left(1-m_{1} f_{C}(0)\right)$, and, hence, negative under the hypothesis. The derivative with respect to $\widetilde{q}$ of (4.1) is proportional to

$$
\left(z+1-f_{C}(0) m_{1}\right)^{2}-f_{C}(0)^{2} m_{1}^{2}+f_{C}(0) m_{1} \rho^{-1}+K f_{C}(0)\left(f_{C}(0) m_{1}-1\right), \quad z=\frac{\tilde{q}}{\tilde{\lambda}} .
$$

Finally, assuming that $\rho \leq 1$, we find three different conditions ensuring that the derivative is positive for any $z \geq 0$.

- For $f_{C}(0) m_{1}<1$,

$$
\frac{K}{m_{1}} \leq \frac{\rho^{-1}-1}{1-m_{1} f_{C}(0)}+\frac{1}{m_{1} f_{C}(0)}
$$

- For $f_{C}(0) m_{1}=1, K \geq 0$.

- For $f_{C}(0) m_{1} \geq 1$,

$$
\frac{K}{m_{1}} \geq \frac{\left(f_{C}(0) m_{1}-\rho^{-1}\right)_{+}}{m_{1} f_{C}(0)-1} .
$$

Moreover, in the $f_{C}(0) m_{1} \geq 1$ case, if we have $K / m_{1}<\left(f_{C}(0) m_{1}-\rho^{-1}\right)_{+} /\left(m_{1} f_{C}(0)-1\right)$ then there are $r_{1}(K) \leq r_{2}(K)$ such that the function $\widetilde{q} \rightarrow f(\widetilde{q}, K)$ defined in $(3)$ is increasing on $\left[0, r_{1}\right]$, decreasing in $\left[r_{1} . r_{2}\right]$, and increasing to $\infty$ on $\left[r_{2}, \infty\right)$. Since the image of $\widetilde{q} \rightarrow f(\widetilde{q}, K)$ is $[0, \infty)$ we can always find an appropriate killing rate, e.g. the smallest solution to $k=f(\widetilde{q}, K)$ (if $k \in \operatorname{Im}_{f}\left(\left[r_{1}, r_{2}\right]\right)$, there are three such solutions, and we can choose the smallest one).

Theorem 1. With claims satisfying $f_{C}(0) m_{1}=1$ (for example, exponential), the readiness function is

$$
f(q)=\frac{(\tilde{\lambda}+\widetilde{q})^{2}-K \tilde{\lambda} \widetilde{q} / m_{1}-\tilde{\lambda} / m_{1}}{\tilde{\lambda} \widetilde{q}}=\frac{\tilde{q}}{\tilde{\lambda}}+\frac{2 m_{1}-K}{m_{1}}+\frac{\tilde{\lambda} m_{1}-1}{m_{1} \tilde{q}} .
$$

Three possibilities arise for subsidiaries when $K=0$.

- The loading condition $\rho<1$ is not satisfied, resulting in rejection.

- The loading condition $\rho<1$ is satisfied, and $k \leq\left((\lambda+q)^{2}-\lambda c \mu\right) / \lambda q$ resulting in acceptance.

- The loading condition $\rho<1$ is satisfied, and $k>\left((\lambda+q)^{2}-\lambda c \mu\right) / \lambda q$, resulting in bailouts up to the level $E\left(r_{i}\right)$, where $r_{i}=\delta(k)-q$, and $\delta(k)$ is the solution of $k=\left((\lambda+\delta)^{2}-\lambda c \mu\right) / \lambda \delta$.

Proof. The derivative of the right-hand side of (4) is proportional to $\delta^{2}+\lambda(\mu c-\lambda), f(\widetilde{q}, K)$ is increasing whenever the loading condition $\rho<1$ is satisfied, and, therefore, an exponential subsidiary not satisfying the efficiency condition (4) can always be rendered efficient by an appropriate killing, increasing its discount rate. 


\section{Hierarchical networks with a deterministic CB: reduction to one dimension}

It turns out that as long as the CB in isolation is a deterministic drift with parameters $u_{0}, c_{0}$, the ruin probability of the $\mathrm{CB}$ is equal to that of a subsidiary with modified parameters

$$
u_{1}^{\prime}=u_{1}+\frac{u_{0}}{k}, \quad c_{1}^{\prime}=c_{1}+\frac{c_{0}}{k},
$$

where $k=k_{1}$, independent of the reset policy. The result is the same as if the CB transfers everything at the time $0_{+}$. This is also the case with several other problems involving a drift CB with no extra liabilities, which ends up liquidated totally.

The pooled assets auxiliary process. Introduce the pooled assets combining $X_{0}$ and the reflected processes $\widetilde{X}_{i}(t)=X_{i}(t)+I_{i}(t)$ in such a way that the transfers and regulation cancel out, that is,

$$
X(t)=X_{0}(t)+\sum_{i=1}^{I} k_{i}\left(X_{i}(t)+I_{i}(t)\right)=u_{0}+c_{0} t+\sum_{i=1}^{I} k_{i} X_{i}(t)=u+c t-\sum_{i=1}^{I} k_{i} S^{(i)}(t)
$$

where we put

$$
u=u_{0}+\sum_{i=1}^{I} k_{i} u_{i}, \quad c=c_{0}+\sum_{i=1}^{I} k_{i} c_{i} .
$$

Remark 6. Note that with spectrally negative Lévy subsidiaries $X(t)$ is also a spectrally negative Lévy process, while $X_{0}(t)$ is a complicated superposition of Sparre Andersen processes.

However, when $I=1$, the ruin time of $X_{0}$ and $U$ coincide. Furthermore, the pooled reserves from the point of view of the subsidiary

$$
\frac{X(t)}{k_{1}}=u_{1}+\frac{u_{0}}{k}+t\left(c_{1}+\frac{c_{0}}{k}\right)-S^{(1)}(t)
$$

has the same law as the subsidiary with combined initial value and income rate.

Remark 7. With several subsidiaries,

$$
\tau=\inf \{t: X(t)<0\}
$$

represents the ruin time if the subsidiaries may start helping each other at no cost, once the $\mathrm{CB}$ is ruined.

These remarks yield the following theorem.

Theorem 2. Let $X_{0}$ be a $C B$ with deterministic drift and subsidiaries with arbitrary structure.

(i) Assume that $I=1$ and put $k=k_{1}$. Then, the ruin time $\tau_{0}$ of the MRN is equal to almost surely, and in distribution, the time $\tau$ of the pooled process defined in (5), and, furthermore, is equal to the ruin time of the subsidiary with modified initial reserve $u_{0} / k+u_{1}$ and premium rate $c_{0} / k+c_{1}, F_{i}(x)$, namely

$$
\Psi(\boldsymbol{u}, \boldsymbol{c}, t)=\Psi_{1}\left(\frac{u_{0}}{k+u_{1}}, \frac{c_{0}}{k+c_{1}, t}\right),
$$

independently of the reset policy. 
(ii) For $I>1$, the time $\tau$ is an upper bound for the ruin time $\tau_{0}$ of the $C B$, that is, $\tau_{0} \leq \tau$.

(iii) Statements similar to (i) hold for any Gerber-Shiu objective, with or without dividends to the subsidiary, as long as the $C B$ is a deterministic drift.

Optimal allocation of total reserves $u_{+}=u_{0}+u_{1}$ and premium rate $c_{+}=c_{0}+c_{1}$. Note that we have the following results.

- When $k \geq 1, u_{0}=c_{0}=0, u_{1}=x, c_{1}=c$ achieve the minimal ruin probability.

- For $k=1$, the ruin probability is independent of the amount $u_{1} \in\left[0, u_{+}\right]$, as well as of the amount of premium rate $c_{1} \in\left[0, c_{+}\right]$.

This optimization result fits the intuitively clear fact that with one subsidiary and no expenses, it is optimal to take advantage of the first transfer without cost to transfer everything to the subsidiary.

The next corollary extends the previous result to a linear chain of the $\mathrm{CB}$, which can represent a chain of reinsurers, see [20].

Corollary 1. Let $X_{0}, \ldots, X_{I-1}$ denote a linear chain of CBs with deterministic drift. Assume that $X_{i}, i=0, \ldots, I-1$ must pay proportional costs $k_{i}$ for bailing out $X_{i+1}$.

Then, the probability of ruin of the MRN satisfies

$\Psi(\boldsymbol{u}, \boldsymbol{c}, t)=\Psi_{I}\left(\frac{u_{0}}{k_{0} \cdots k_{I-1}}+\frac{u_{1}}{k_{1} \cdots k_{I-1}}+\cdots u_{I}, \frac{c_{0}}{k_{0} \cdots k_{I-1}}+\frac{c_{1}}{k_{1} \cdots k_{I-1}}+\cdots c_{I}, t\right)$,

where $\Psi_{I}$ is the ruin probability of the last subsidiary in the chain.

Remark 8. One interesting feature of this result is that it does not require any assumption on the probabilistic structure of the subsidiary risk process.

Another interesting feature is that similar reductions hold for other problems, as long as $I=1$ and the main branch is a deterministic drift (in the absence of subsidiaries). For example, if $V$ denotes the value function of the de Finetti objective, we also have

$$
V(\boldsymbol{u}, \boldsymbol{c})=V_{1}\left(\frac{u_{0}}{k+u_{1}}, \frac{c_{0}}{k+c_{1}}\right) .
$$

One may add ruin observed only at Poissonian times, Parisian ruin, etc.

\section{SNMAP approximations of a nondeterministic CB}

In this section we turn to the case of a nondeterministic CB with one subsidiary. We have seen in the previous sections that the risk process of the CB is itself a Sparre Andersen process in which the interarrival times are the first-passage times and respectively the bailout amounts for the subsidiary. We provide here a methodology to arrive at an SNMAP approximation for the $\mathrm{CB}$ risk process, with the goal to provide an input to the available scale methodology toolbox (see [21]-[23], [26]) which can be used to simultaneously solve a wide variety of insurance problems for this $\mathrm{CB}$ risk process. We exemplify in this section with the computation of the optimal dividend barrier for the $\mathrm{CB}$ itself, and implicitly for the whole network. 


\subsection{Two point Padé approximations for the downward ladder time of the Cramér- Lundberg process with exponential claims}

In this subsection we consider the particular case when the subsidiary risk process follows a Cramér-Lundberg process with exponential claims and we are interested in its downward ladder time.

Our approach is based on the idea that approximating the excursions of a process ensures approximating the process, and, in particular, various functionals of the process [27], [34].

With phase-type jumps, one would need to provide bivariate matrix-exponential approximations for the joint law of the downward ladder time and height of a Sparre Andersen process.

In the case of exponential claims, the density of the downward ladder time may be expressed as a hypergeometric function, that is,

$$
\rho(t)=\widetilde{\rho}(c \mu t) c \mu, \quad \widetilde{\rho}(t)=\rho \mathrm{e}^{-(1+\rho) t}{ }_{0} F_{1}\left(2, \rho t^{2}\right) .
$$

However, what we need is a phase-type approximation of this. This topic has already been considered in [1] (at order-2), as one of many possible methods for approximating the M/M/1 busy period density.

We recall now some basic facts on this case, see [6].

Lemma 2. (i) With Poisson arrivals of rate $\lambda$ and exponential claims of rate $\mu$, the Laplace transform of the downward ladder time density satisfies a quadratic equation

$$
\hat{\rho}_{q}=\widehat{a} q+c \mu-c \mu \hat{\rho}_{\delta}=\frac{\lambda}{\lambda+q+c \mu-c \mu \hat{\rho}_{\delta}}=\frac{\rho}{1+\delta+\rho-\hat{\rho}_{\delta}}, \quad \rho:=\frac{\lambda}{c \mu}, \quad \delta=\frac{q}{c \mu}
$$

with solution

$$
\hat{\rho}_{\delta}=\widehat{\widetilde{\rho}}_{\delta}=\frac{1}{2}\left(\delta+\rho+1-\sqrt{(\delta+\rho+1)^{2}-4 \rho}\right) .
$$

(ii) The Laplace transform (6) may be computed iteratively by the continued fraction expansion

$$
\widehat{\widetilde{\rho}}_{\delta}=\rho\left[1+\delta+\rho-\frac{\rho}{1+\delta+\rho-\rho /(1+\delta+\rho-s \ldots)}\right]^{-1} .
$$

The convergents $\widehat{\widetilde{\rho}}_{\delta}^{(n)}, n=1,2,3, \ldots$, with s constant satisfy

$$
\widehat{\widetilde{\rho}}_{\delta}^{(n)}=\frac{\rho P_{n-1}(\delta+\rho+1)}{P_{n}(\delta+\rho+1)}
$$

[31, Equation (75)], where $P_{n}(x)$ are Chebyshev polynomials [31, Equation (77)]. When $s=0$, the first three are

$$
\left\{\frac{\rho}{\delta+\rho+1}, \frac{\rho(\delta+\rho+1)}{\delta^{2}+2 \rho \delta+2 \delta+\rho^{2}+\rho+1}, \frac{\rho\left(\delta^{2}+2 \rho \delta+2 \delta+\rho^{2}+\rho+1\right)}{(\delta+\rho+1)\left(\delta^{2}+2 \rho \delta+2 \delta+\rho^{2}+1\right)}, \ldots\right\} .
$$

Decomposing as a partial fraction the 3 convergent yields an order-3 rational approximation of the Laplace transform of the ladder time, that is,

$$
\begin{aligned}
\hat{\rho}_{\delta} & \approx \frac{\rho}{\delta+\rho+1} \frac{(\delta+\rho+1)^{2}-\rho}{(\delta+\rho+1)^{2}-2 \rho} \\
& =\frac{\rho}{2}\left(\frac{1}{\delta+\rho+1}+\frac{1 / 2}{\delta+\rho+1+\sqrt{2 \rho}}+\frac{1 / 2}{\delta+\rho+1-\sqrt{2 \rho}}\right) .
\end{aligned}
$$


Inverting the Laplace transform yields a hyperexponential density approximation

$$
\begin{aligned}
\tilde{\rho}(t) & \approx \rho \mathrm{e}^{-(1+\rho) t}\left(\frac{1}{2}+\frac{1}{4}\left(\mathrm{e}^{-\sqrt{2 \rho} t}+\mathrm{e}^{+\sqrt{2 \rho} t}\right)\right) \\
& :=\sum_{i=0}^{2} \alpha_{i} \lambda_{i} \mathrm{e}^{-\lambda_{i} t}=\frac{\rho}{2} \mathrm{e}^{-(1+\rho) t}+\frac{\rho}{4} \mathrm{e}^{-(1+\rho+\sqrt{2 \rho}) t}+\frac{\rho}{4} \mathrm{e}^{-(1+\rho-\sqrt{2 \rho}) t},
\end{aligned}
$$

where $\alpha_{0}=\rho / 2(1+\rho)$ and $\alpha_{1,2}=\rho / 4(1+\rho \pm \sqrt{2 \rho})$ are nonnegative for any $\rho \in[0, \infty)$. Furthermore, $\sum_{i} \alpha_{i}<\frac{3}{4} \rho$ if and only if $\rho<1$, thus providing us with a valid approximation for any $\rho$ in this range.

Remark 9. A further simplification of the Laplace transform (6) may be obtained by factoring $\delta+\rho+1$ and changing variables $a=\rho(1+\rho+\delta)^{-2}$, thus

$$
\hat{\rho}_{\delta}=\widehat{\widetilde{\rho}}_{\delta}=\frac{\delta+\rho+1}{2}\left(1-\sqrt{1-4 \frac{\rho}{(\delta+\rho+1)^{2}}}\right)=\frac{\rho}{\delta+\rho+1} \frac{1-\sqrt{1-4 a}}{2 a} .
$$

The second factor is the generating function of the famous Catalan numbers

$$
\frac{1-\sqrt{1-4 a}}{2 a}=\sum_{k=0}^{\infty} \frac{\left(\begin{array}{c}
2 k \\
k
\end{array}\right)}{k+1} a^{k}=1+a+2 a^{2}+5 a^{3}+14 a^{4}+\cdots
$$

and a continued fraction representation

$$
\frac{1-\sqrt{1-4 a}}{2 a}=\left[1-\frac{a}{1-a /(1+\cdots)}\right]^{-1}
$$

may be found, e.g. in [16, Equation (7.7.5)]. As is well known, the Padé approximations obtained by truncating continued fractions have good properties, such as larger domains of convergence than the corresponding power series. The lowest order approximations of (8) are

$$
\frac{1}{1-a}, \quad \frac{1-a}{1-2 a}, \quad \frac{1-2 a}{1-3 a+a^{2}}, \quad \frac{1-3 a+a^{2}}{1-4 a+3 a^{2}}, \quad \frac{1-4 a+3 a^{2}}{1-5 a+6 a^{2}-a^{3}}, \ldots
$$

Lemma 3. The rational convergents $R_{n}$ of the continued fraction (8) increase towards (1 $\sqrt{1-4 a}) / 2 a$ for all $a \in\left(0, \frac{1}{4}\right)$.

Proof. This is immediate by the positivity of $a$.

Remark 10. Alternatively, we may use two-point Padé approximations which ensure also the equality of the derivatives around 0 . Note that [1, Section 3] provides an in-depth numerical comparison of several hyper-exponential approximations of order-2, and it is seen that fitting the derivatives yields excellent results around 0, while fitting the moments is less satisfactory, since better results may be obtained with asymptotic approximations.

Let us now invert the lowest order two-point Padé approximation of $\hat{\rho}(\delta)$ which ensures also the condition $\tilde{\hat{\rho}}_{\delta=0}=\rho, \rho \in(0,1]$, that is,

$$
\begin{aligned}
& \frac{\rho\left(\delta^{2}+2 \rho \delta+2 \delta+\rho+1\right)}{(\delta+\rho+1)\left(\delta^{2}+2 \rho \delta+2 \delta+1\right)} \\
& \quad=\frac{\rho}{\rho+2}\left(\frac{\rho+1}{\delta+\rho+1}+\frac{\delta+\rho+1}{\delta^{2}+2 \delta(\rho+1)+1}\right) \\
& \quad=\frac{\rho}{\rho+2}\left(\frac{\rho+1}{\delta+\rho+1}+\frac{1}{\lambda_{1}-\lambda_{2}}\left(\frac{\lambda_{1}-(\rho+1)}{\delta+\lambda_{1}}+\frac{\rho+1-\lambda_{2}}{\delta+\lambda_{2}}\right)\right)
\end{aligned}
$$


and

$$
\lambda_{1,2}=\rho+1 \pm \sqrt{\rho(\rho+2)} .
$$

This yields a density approximation

$$
\widetilde{\rho}(t) \approx \sum_{i=0}^{2} \alpha_{i} \lambda_{i} \mathrm{e}^{-\lambda_{i} t}
$$

where

$$
\begin{gathered}
\alpha_{0}=\frac{\rho}{\rho+2}, \quad \alpha_{1}=\frac{\rho}{\rho+2} \frac{1-(\rho+1) / \lambda_{1}}{\lambda_{1}-\lambda_{2}}=\frac{\rho^{1 / 2}(\sqrt{\rho(\rho+2)}+1)}{2(\rho+2)^{3 / 2}(\rho+1+\sqrt{\rho(\rho+2)})}, \\
\alpha_{2}=\frac{\rho}{\rho+2} \frac{(\rho+1) / \lambda_{2}-1}{\lambda_{1}-\lambda_{2}}=\frac{\rho^{1 / 2}(\sqrt{\rho(\rho+2)}-1)}{2(\rho+2)^{3 / 2}(\rho+1-\sqrt{\rho(\rho+2)})} .
\end{gathered}
$$

Note that $\alpha_{i}$ are nonnegative for any $\rho \in[0, \infty)$, and $\sum_{i} \alpha_{i}=\rho$. See Figures 1 and 2 .

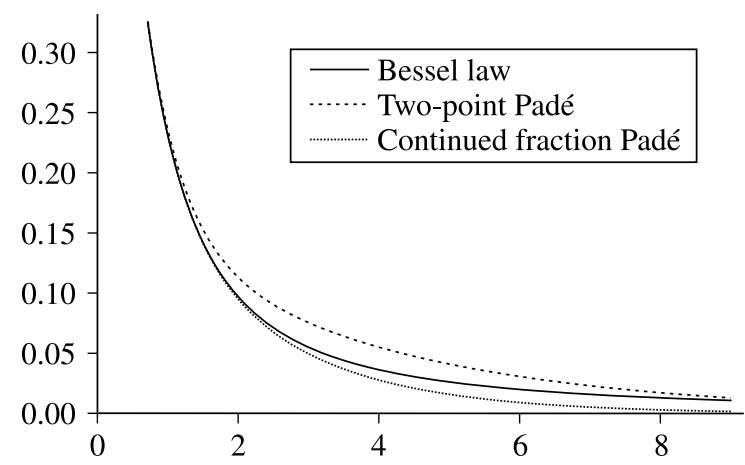

Figure 1: Two order-3 matrix exponential approximations (7), (9) of $\rho(t)=\psi(t ; 0)$ with $\lambda=\mu=1$, $\rho=\frac{7}{8}=c^{-1}$. The Bessel law is in between the two-point Padé approximation which is tight both at 0 and $\infty$, and the classic continued fraction Padé approximation, which is better near 0 .

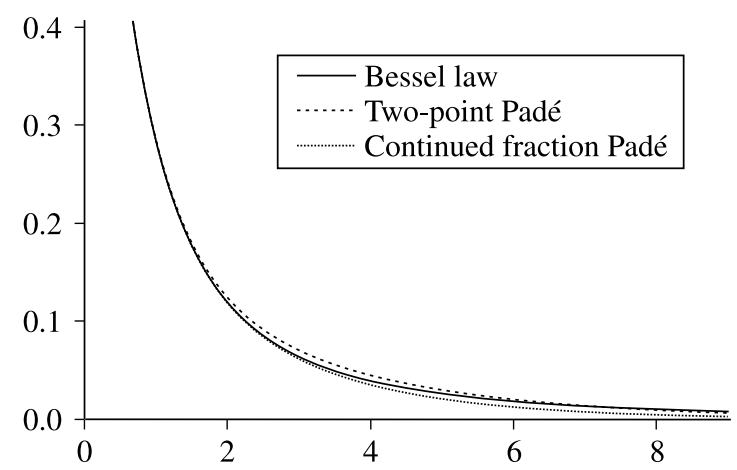

Figure 2: Two order-3 matrix exponential approximations (7), (9) of $\psi(t ; 0)$ with $\lambda=\mu=1, \rho=\frac{1}{2}=$ $c^{-1}$. The fit is better as $\rho$ gets further from 1 , and could be improved by using a combination of the two approximations, of order-5. 


\subsection{Padé-based SNMAP approximations for $X(t)$ when subsidiary claims are exponential}

Having established in the previous subsection convenient approximations for the subsidiary risk process, we now approximate the $\mathrm{CB}$ risk process.

Note that even though finite-time ruin probabilities have an explicit Bessel density with exponential claims, we will replace them by matrix exponential approximations, since this allows solving network problems by the SNMAP methodology.

After applying the order-3 approximation (9) to the subsidiary's ladder time, the CB becomes a MAP with three states, with transition rates

$$
Q_{i j}=\lambda_{i} \alpha_{j}
$$

accompanied by exponential jumps of rate $\mu / k$ translated by $K$ (we could include here phasetype jumps to the $\mathrm{CB}$, and fixed costs, since these pose no problem to the MAP methodology).

When $K=0$,

$$
\widehat{f}(s)=\frac{\mu / k}{s+\mu / k},
$$

and we find from the general formula that the symbol of the approximated CB is

$$
\begin{aligned}
\mathbb{K}(s) & =\operatorname{diag}\left(c_{0} s-\lambda_{i}\right)+\lambda \vec{\alpha} \widehat{f}(s) \\
& =\left(\begin{array}{ccc}
c_{0} s-\lambda_{0}+\lambda_{0} \alpha_{0} \frac{\mu / k}{s+\mu / k} & \lambda_{0} \alpha_{1} \frac{\mu / k}{s+\mu / k} & \lambda_{0} \alpha_{2} \frac{\mu / k}{s+\mu / k} \\
\lambda_{1} \alpha_{0} \frac{\mu / k}{s+\mu / k} & c_{0} s-\lambda_{1}+\lambda_{1} \alpha_{1} \frac{\mu / k}{s+\mu / k} & \lambda_{1} \alpha_{2} \frac{\mu / k}{s+\mu / k} \\
\lambda_{2} \alpha_{0} \frac{\mu / k}{s+\mu / k} & \lambda_{2} \alpha_{1} \frac{\mu / k}{s+\mu / k} & c_{0} s-\lambda_{2}+\lambda_{2} \alpha_{2} \frac{\mu / k}{s+\mu / k}
\end{array}\right) .
\end{aligned}
$$

To apply the scale-based MAP methodology, it is convenient to transform this MAP with exponential jumps into a continuous MMBM

$$
\widetilde{\mathbb{K}}(s)=\left(\begin{array}{cccccc}
c_{0} s-\lambda_{0} & 0 & 0 & \lambda_{0} \alpha_{0} & \lambda_{0} \alpha_{1} & \lambda_{0} \alpha_{2} \\
0 & c_{0} s-\lambda_{1} & 0 & \lambda_{1} \alpha_{0} & \lambda_{1} \alpha_{1} & \lambda_{1} \alpha_{2} \\
0 & 0 & c_{0} s-\lambda_{2} & \lambda_{2} \alpha_{0} & \lambda_{2} \alpha_{1} & \lambda_{2} \alpha_{2} \\
\frac{\mu}{k} & 0 & 0 & -s-\frac{\mu}{k} & 0 & 0 \\
0 & \frac{\mu}{k} & 0 & 0 & -s-\frac{\mu}{k} & 0 \\
0 & 0 & \frac{\mu}{k} & 0 & 0 & -s-\frac{\mu}{k}
\end{array}\right) .
$$

After obtaining an SNMAP approximation for $X(t)$, we may solve approximatively various problems related to this process, using the package [22].

We illustrate this by finding, in Figure 3, the optimal dividend barrier. It is beyond the scope of this paper to investigate further this problem in particular (which deserves a separate treatment), as our main goal was not the solution of the problem per se, but to provide an adequate input to the toolbox that solves this type of problems. 


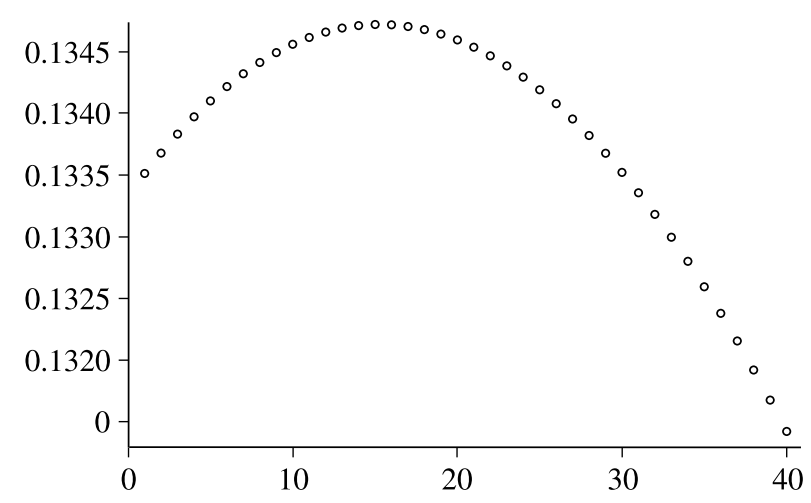

FIGURE 3: Dividends as a function of barrier, starting from $u_{0}=u_{1}=0$, with $\lambda=\mu=k=1, c_{1}=$ $4, c_{0}=24$, obtained by the scale matrix methodology [21]. The maximizing barrier is approximately 0.17 .

\section{Acknowledgements}

We thank H. Albrecher, B. Avanzi, L. Breuer, E. Frostig, J. Ivanovs, R. Loeffen, M. Pistorius, and T. Rolski for useful discussions.

\section{References}

[1] Aвate, J. And Whitt, W. (1988). Approximations for the M/M/1 busy-period distribution. In Queueing Theory and Its Applications (CWI Monogr. 7), North-Holland, Amsterdam, pp. 149-191.

[2] Albrecher, H., Hartinger, J. and Thonhauser, S. (2007). On exact solutions for dividend strategies of threshold and linear barrier type in a Sparre Andersen model. ASTIN Bull. 37, 203-233.

[3] Asmussen, S. (2003). Applied Probability and Queues (Appl. Math. 51). Springer, New York.

[4] Asmussen, S. and Albrecher, H. (2010). Ruin Probabilities (Adv. Ser. Statist. Sci. Appl. Prob. 14), 2 nd edn. World Scientific, Hackensack, NJ.

[5] Avanzi, B. (2009). Strategies for dividend distribution: a review. North Amer. Actuarial J. 13, 217-251.

[6] Avram, F. And Minca, A. (2015). Steps towards a management toolkit for central branch risk networks, using rational approximations and matrix scale functions. In Modern Trends in Controlled Stochastic Processes: Theory and Applications, Luniver Press, Bristol.

[7] Avram, F., Kyprianou, A. E. And Pistorius, M. R. (2004). Exit problems for spectrally negative Lévy processes and applications to (Canadized) Russian options. Ann. Appl. Prob. 14, 215-238.

[8] Avram, F., Palmowski, Z. and Pistorius, M. R. (2007). On the optimal dividend problem for a spectrally negative Lévy process. Ann. Appl. Prob. 17, 156-180.

[9] Avram, F., Palmowski, Z. and Pistorius, M. R. (2008). Exit problem of a two-dimensional risk process from the quadrant: exact and asymptotic results. Ann. Appl. Prob. 18, 2421-2449.

[10] Avram, F., Palmowski, Z. and Pistorius, M. (2009). On optimal dividend distribution for a Cramér-Lundberg process with exponential jumps in the presence of a linear Gerber-Shiu penalty function. Monogr. Semin. Mat. García Galdeano nn 1, 10.

[11] Avram, F., Palmowski, Z. and Pistorius, M. R. (2015). On Gerber-Shiu functions and optimal dividend distribution for a Lévy risk process in the presence of a penalty function. Ann. Appl. Prob. 25, 1868-1935.

[12] Azcue, P. and Muler, N. (2005). Optimal reinsurance and dividend distribution policies in the CramérLundberg model. Math. Finance 15, 261-308.

[13] Badescu, A. L., Cheung, E. C. K. and Rabehasaina, L. (2011). A two-dimensional risk model with proportional reinsurance. J. Appl. Prob. 48, 749-765.

[14] Bertoin, J. (1998). Lévy Processes (Camb. Tracts Math. 121). Cambridge University Press.

[15] Chan, W.-S., Yang, H. and Zhang, L. (2003). Some results on ruin probabilities in a two-dimensional risk model. Insurance Math. Econom. 32, 345-358.

[16] CuYt, A. et al. (2008). Handbook of Continued Fractions for Special Functions. Springer, New York.

[17] Czarna, I. and Renaud, J.-F. (2015). A note on Parisian ruin with an ultimate bankruptcy level for Lévy insurance risk processes. Preprint.

[18] Eisenberg, J. AND Schmidli, H. (2011). Minimising expected discounted capital injections by reinsurance in a classical risk model. Scand. Actuarial J. 2011, 155-176. 
[19] Gerber, H. U. (1972). Games of economic survival with discrete-and continuous-income processes. Operat. Res. 20, 37-45.

[20] Gerber, H. U. (1984). Chains of reinsurance. Insurance Math. Econom. 3, 43-48.

[21] Ivanovs, J. (2011). One-sided Markov additive processes and related exit problems. Doctoral Thesis.

[22] Ivanovs, J. (2013). Spectrally-negative Markov additive processes: in continuous time, version 1.0. Mathematica 8 package. Available at https://sites.google.com/site/jevgenijsivanovs/files.

[23] Ivanovs, J. AND Palmowski, Z. (2012). Occupation densities in solving exit problems for Markov additive processes and their reflections. Stoch. Process. Appl. 122, 3342-3360.

[24] Kulenko, N. And Schmidli, H. (2008). Optimal dividend strategies in a Cramér-Lundberg model with capital injections. Insurance Math. Econom. 43, 270-278.

[25] Kuznetsov, A., Kyprianou, A. E. And Rivero, V. (2013). The theory of scale functions for spectrally negative Lévy processes. In Lévy Matters II (Lecture Notes Math. 2061), Springer, Berlin, pp. 97-186.

[26] Kyprianou, A. E. AND PalmowsKi, Z. (2008). Fluctuations of spectrally negative Markov additive processes. In Séminaire de Probabilités XLI (Lecture Notes Math. 1934), Springer, Berlin, pp. 121-135.

[27] Lambert, A., Simatos, F. And Zwart, B. (2013). Scaling limits via excursion theory: interplay between CrumpMode-Jagers branching processes and processor-sharing queues. Ann. Appl. Prob. 23, 2357-2381.

[28] Loeffen, R. L. (2008). On optimality of the barrier strategy in de Finetti's dividend problem for spectrally negative Lévy processes. Ann. Appl. Prob. 18, 1669-1680.

[29] Loeffen, R. L. And Renaud, J.-F. (2010). De Finetti's optimal dividends problem with an affine penalty function at ruin. Insurance Math. Econom. 46, 98-108.

[30] LøKKa, A. AND Zervos, M. (2008). Optimal dividend and issuance of equity policies in the presence of proportional costs. Insurance Math. Econom. 42, 954-961.

[31] Neuts, M. (1969). The queue with Poisson input and general service times, treated as a branching process. Duke Math. J. 36, 215-231.

[32] Shreve, S. E., Lehoczky, J. P. and Gaver, D. P. (1984). Optimal consumption for general diffusions with absorbing and reflecting barriers. SIAM J. Control Optimization 22, 55-75.

[33] Von Dahlen, S. And Von Peter, G. (2012). Natural catastrophes and global reinsurance-exploring the linkages. BIS Quart. Rev. 23-35.

[34] Yano, K. (2014). Functional limit theorems for processes pieced together from excursions. Preprint. Available at https://arXiv.org/abs/1309.2652v3. 